BACTERIAL PATHOGENESIS

\section{Rewiring cellular dynamics and metabolism}

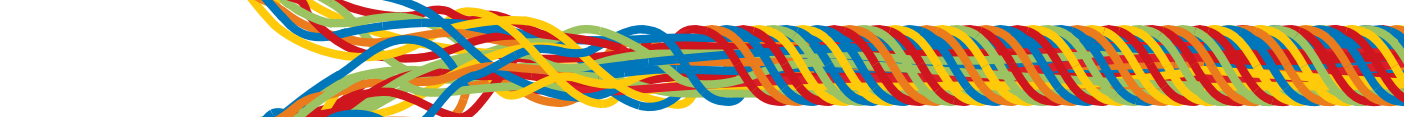

Legionella pneumophila, the causative agent of Legionnaires' disease, is an opportunistic pathogen that establishes a replicative niche, termed the Legionella-containing vacuole (LCV), within human lung macrophages. L. pneumophila injects hundreds of bacterial effector proteins into the host cytoplasm through its type IV secretion system (T4SS) to subvert host processes and promote intracellular replication. It was previously reported that the LCV is rapidly surrounded by mitochondria, and it has been suggested that the host organelles are recruited by T4SS effectors; however, neither the bacterial T4SS effectors implicated in mitochondrial recruitment nor the mitochondrial functions likely to be modulated by L. pneumophila have been identified. In this study, Escoll et al. report that L. pneumophila regulates mitochondrial dynamics to subvert the mitochondrial bioenergetics of infected cells in a process that involves a bacterial T4SS effector and a host fission protein.

First, time-lapse confocal microscopy analysis of infected human monocyte-derived macrophages showed that the LCV is rapidly (that is, $1-6 \mathrm{~h}$ post-infection) surrounded by mitochondria, thus confirming earlier reports in fixed cells. The experiments also showed that the interactions between mitochondria and the LCV were highly dynamic and transient. Interestingly, when the authors monitored mitochondrial recruitment in cells infected with L. pneumophila mutants that lacked a functional T4SS, they found no difference compared with the ones infected with wild-type bacteria, which suggests that mitochondria surround the LCV independent of T4SS effectors.

Moreover, L. pneumophila induced mitochondrial fragmentation during the first $6 \mathrm{~h}$ of infection. This effect was dependent on the T4SS, as cells that were infected with mutant bacteria that lacked the secretion system showed decreased mitochondrial fragmentation compared with cells infected with wild-type bacteria. Next, when the authors infected macrophages with different $L$. pneumophila mutants lacking specific T4SS effectors they found that one effector mutant did not induce mitochondrial fragmentation in infected cells; accordingly, the authors named this effector protein mitochondrial fragmentation factor (MitF). Further elucidating the mechanisms involved in mitochondrial fragmentation induced by MitF, they showed that the host fission factor dynamin 1-like protein (DNM1L, also known as DRP1) accumulates on host mitochondria during infection with L. pneumophila in a T4SS-dependent manner. Moreover, DNM1L-induced mitochondrial fragmentation was required for intracellular replication of L. pneumophila, which suggests that the bacteria subvert organelle dynamics to promote their survival. However, fragmentation of mitochondria is considered to be a hallmark of cell death, which, in turn, is a defence mechanism against pathogens. Although the authors of this study observed DNM1L recruitment to the mitochondria of infected cells, early or late signs of cell death were lacking, including the release of cytochrome $c$ or caspase activation. Thus, they suggest that during L. pneumophila infection mitochondrial fission is a L. pneumophilainduced cellular event that is segregated from cell death pathways.

Finally, as changes in mitochondrial morphology are thought to be linked to changes in the bioenergetics of the cell, the authors investigated whether infection with L. pneumophila has an effect on mitochondrial respiration. Indeed, they were able to show that $1 \mathrm{~h}$ post-infection L. pneumophila progressively induces Warburg-like metabolism in the cell (that is, characterized by the metabolic switch from oxidative phosphorylation to aerobic glycolysis) by a mechanism that is dependent on a functional T4SS and involves DNM1L.

In summary, the results from this study suggest that the bacteriainduced modulation of mitochondrial dynamics during infection affects host metabolism and represents a key virulence strategy used by L. pneumophila.

Andrea Du Toit

ORIGINAL ARTICLE Escoll, P. et al. Legionella pneumophila modulates mitochondrial dynamics to trigger metabolic repurposing of infected macrophages. Cell Host Microbe http://dx.doi. org/10.1016/j.chom.2017.07.020 (2017) FURTHER READING Qiu, J. \& Luo, Z.-Q. Legionella and Coxiella effectors: strength in diversity and activity. Nat. Rev. Microbiol. http://dx.doi. org/10.1038/nrmicro.2017.67 (2017) 